\title{
ARRHENIUS JUMP RATE OF MUONS IN METALS AND METALLIC COMPOUNDS
}

\author{
G.C. Mainato \\ R.K.M.V.C. College, Rahara, 24 Parganas (North), West Bengal, India
}

(Received March 15, 1994)

\begin{abstract}
Using the energy loss rate in the process $\mu^{+}+\mathrm{e}^{-} \rightarrow \nu_{\mathrm{e}}+\bar{\nu}_{\mu}$ we develop a model which formulates the jump rate for muon diflusion in metals platinum, lead and metallic compounds $\mathrm{ZrV}_{2}, \mathrm{HI}_{2} \mathrm{Co}$ and $\mathrm{V}_{3} \mathrm{Si}$. The jump rate is found to follow an Arrhenius-like relation over some temperature ranges.
\end{abstract}

PACS numbers: $66.30 . \mathrm{Jt}$

\section{Introduction}

In our earlier papers [1-5] diffusion of muons in metals and non-stoichiometric metallic and intermetallic hydrides has been studied in a newly developed model which formulates the jump rate for muon diffusion in an Arrhenius form. The model considers the muons, upon being incident on the target, can diffuse through an electron gas of the target. While diffusing through the electron gas the muons find electrons to collide and result in an annihilation process in which energy is liberated from the target due to neutrino-antineutrino emission. This energy loss rate is made related to the jump rate for muon diffusion in all these targets.

This study of muon diffusion is readily extended to the case of some metallic compounds which are used as good targets. Therefore this article becomes an extension of our previous works and presents some more results for the jump rate of muons in metals like platinum and lead and some good metallic compounds like $\mathrm{ZrV}_{2}, \mathrm{Hf}_{2} \mathrm{Co}$ and $\mathrm{V}_{3} \mathrm{Si}$.

\section{Theory}

A wide beam of polarized and highly energetic muons, incident upon the target, is assumed to form a "muon gas" which diffuses through an "electron gas" offered by the target material. In case of metallic and metallic compound-targets the electron gas is formed by the valence electrons of the targets. For electron densities found in metals, the Coloumb potential of the muon is reduced by screening to the extent that a bound state with any individual electron is quite impossible [6-8] so it is assumed that in these targets the incident muon does not form a muonium atom and behaves as if it was a free particle. 
During the diffusion through the electron gas a muon visits many sites by performing jumps and finally disappeares in a muon-electron annihilation process represented by

$$
\mu^{+}+\mathrm{e}^{-} \rightarrow \nu_{\mathrm{e}}+\bar{\nu}_{\mu} .
$$

It is further assumed that the energy necessary to produce such an annihilation process corresponds to the activation energy for the diffusion of muon through the electron gas.

While jumping over the intervening barriers through the electron gas the muon finds an electron to collide and results in the annihilation process. The jump rate for muon diffusion becomes proportional to the energy loss rate which is liberated from the target due to neutrino-antincutrino emission. In such a picture, the jump rate for diffusion is written in the form

$$
\frac{1}{\tau}=F(z) \frac{\mathrm{d} U}{\mathrm{~d} t} \quad\left[\mathrm{~s}^{-1}\right],
$$

where $\mathrm{d} U / \mathrm{d} l$ represents the energy loss rate libcrated from the target, $F(z)$ denotes a constant of proportionality depending upon the target only.

The energy loss rate is given by

$$
-\frac{\mathrm{d} U}{\mathrm{~d} t}=\left(E_{\mu_{+}}+E_{-}\right) \sigma n_{+} n_{-} v_{\text {rel }} \quad\left[\mathrm{erg} \mathrm{cm}^{-3} \mathrm{~s}^{-1}\right],
$$

where $E_{\mu^{+}}\left(E_{-}\right)$represents the total energy of the muon (electron), $n_{+}\left(n_{-}\right)$ represents the number density of the muon (electron), $v_{\text {rel }}$ is the relative velocity between the muon and the electron in the electron gas and $\sigma$ is the cross-section of the reaction (1). The cross-section of the reaction is calculated by using a standard ?lectroweak theory [9] and is found to be [1-5]:

$$
\sigma=1.7 \times 10^{-40} \quad\left[\mathrm{~cm}^{2}\right] .
$$

The number density of muon is given by

$$
n_{+}=1.4 \times 10^{19} T^{3 / 2} \mathrm{e}^{-E / T} \quad\left[\mathrm{~cm}^{-3}\right] .
$$

IIere $E$ represents an energy necessary to produce the annihilation process and is assumed to be equal to the activation energy for the diffusion process.

The number density of electron is given by

$$
n_{-}=\frac{N d v}{M} \quad\left[\mathrm{~cm}^{-3}\right] \text {. }
$$

Here $v$ represents the total number of valence electrons present in one molecule of the target of density $d$ and molecular weight $M, N$ is the Avogadro number. For metallic targets the molecular weight $M$ is replaced by the atomic weight $A$.

For an over-barrier diffusion of muons in metals the function $F(z)$ is suggested to have an empirical form [1-5]:

$$
F(z)=\frac{N A}{z^{2} d c^{2}} \quad\left[\operatorname{erg}^{-1} \mathrm{~cm}^{3}\right],
$$

where $z$ represents the atomic number of the metal. For metallic compunds the function has the form

$$
F(z)=\frac{N M}{z^{2} d c^{2}} \quad\left[\mathrm{erg}^{-1} \mathrm{~cm}^{3}\right] .
$$


IIere $z$ represents the sum of the atomic numbers of the atoms present in one molecule of the metallic compound.

For an under-barrier tunneling the function $F(z)$ is multiplied by the potential barrier transparency coefficient $\beta$ given by [10]:

$$
\beta=\exp \left[-\frac{2}{h}\left(2 m_{\mu} H\right)^{1 / 2} b\right] .
$$

IIere $H$ and $b$ represent respectively the height and breadth of the potential barrier.

Therefore finally the jump rate for muon diffusion in an over-barrier diffusion process is

$$
\frac{1}{\tau}=\frac{6.8 \times 10^{12}}{z^{2}} v T^{3 / 2} \mathrm{e}^{-E / T} \quad\left[\mathrm{~s}^{-1}\right] .
$$

For an under-barrier tunelling motion the jump rate formula is

$$
\frac{1}{\tau}=\frac{6.8 \times 10^{12}}{z^{2}} v \beta T^{3 / 2} \mathrm{e}^{-E / T} \quad\left[\mathrm{~s}^{-1}\right] .
$$

\section{Results}

The present theory is now applied to the case of metals: platinum, lead, and metallic compounds: $\mathrm{ZrV}_{2}, \mathrm{Hf}_{3} \mathrm{Co}$ and $\mathrm{V}_{3} \mathrm{Si}$. The activation energy for muon diffusion is used from the respective experimental results.

\subsection{Platinum}

Muon diffusion in platinum was studicd over the temperature range $4-300 \mathrm{~K}$ $[11,12]$. Muons are observed stationary below $100 \mathrm{~K}$ above which the muons diffuse by a tunnelling process. Over $100-220 \mathrm{~K}$ the under-barrier jump rate for muon diffusion is described by an Arrhenius relation

$$
\frac{1}{\tau}=\nu_{0} \mathrm{e}^{-E / T} \quad\left[\mathrm{~s}^{-1}\right]
$$

with $\nu_{0}=10^{8.9(3)} \mathrm{s}^{-1}$ and $E=1080 \mathrm{~K}$.

In the present model muon is assumed to tunnel with an activation energy $1000 \mathrm{~K}$. Then using the barrier width $b=2 \AA$ the quantum barrier transparency coefficient $\beta$ becomes $1.8 \times 10^{-4}$ and the under-barrier jump rate for muon diffusion is given by

$$
\frac{1}{\tau}=4 \times 10^{5} T^{3 / 2} \mathrm{e}^{-1000 / T} \quad\left[\mathrm{~s}^{-1}\right] .
$$

This formula agrees with the experimental results over the temperature range $100-220 \mathrm{~K}$.

\subsection{Lead}

In lead muons are static up to $40 \mathrm{~K}[11,12]$; over the temperature range $40-100 \mathrm{~K}$ lead shows very fast muon diffusion with a small activation energy $\approx 250 \mathrm{~K}$. 
In the present model muon is assumed to tunnel with an activation energy $250 \mathrm{~K}$. For $b=4.3 \AA, \beta$ becomes $9.2 \times 10^{-5}$ and the under-barricr jump rate for muon diffusion is given by

$$
\frac{1}{\tau}=3.7 \times 10^{5} T^{3 / 2} \mathrm{e}^{-250 / T} \quad\left[\mathrm{~s}^{-1}\right] .
$$

\section{3. $\mathrm{ZrV}_{2}$}

In this alloy muon is found [13] static up to $50 \mathrm{~K}$. For $T>50 \mathrm{~K}$, the muon diffuses in a tunnelling process and over $50-250 \mathrm{~K}$ the under-barrier jump rate for muon diffusion is described by the formula

$$
\frac{1}{\tau}=(1.8 \pm 0.4) \times 10^{7} \exp \left[-\frac{(20 \pm 2) \mathrm{meV}}{k_{\mathrm{B}} T}\right] \quad\left[\mathrm{s}^{-1}\right] .
$$

This result can be explained by the present theory which assumes the tunnelling of muon under a potential barrier $(H)$. Using the potential barrier for hydrogen diffusion $\left(H_{\mathrm{p}}\right)=1000 \mathrm{~K}[14]$ and considering the isotopic reason, $H$ is set equal to $1000 \mathrm{~K}$. Then for $b=3 \AA, \beta$ becomes $2.3 \times 10^{-6}$. With an activation energy $20 \mathrm{meV}$, the under-barrier jump rate for muon diffusion is given by

$$
\frac{1}{\tau}=1.3 \times 10^{4} T^{3 / 2} \exp \left(-\frac{20 \mathrm{meV}}{k_{\mathrm{B}} T}\right) \quad\left[\mathrm{s}^{-1}\right] .
$$

This formula agrees well with the experimental values over the temperature range $50-250 \mathrm{~K}$.

\section{4. $\mathrm{II}_{2} \mathrm{Co}$}

Diffusion of muon in highly pure $\mathrm{II}_{2}$ Co was studied up to room temperature by Baudry et al. [15]. Muons were found to be localized at different interstitial positions below $200 \mathrm{~K}$ over which the muons diffuse in a tunnelling process. Over $220-300 \mathrm{~K}$ the jump rate for muon diffusion is described by the relation

$$
\frac{1}{\tau}=4.5(3) \times 10^{8} \exp \left[-\frac{(190 \pm 5) \mathrm{meV}}{k_{\mathrm{B}} T}\right] \quad\left[\mathrm{s}^{-1}\right] .
$$

In the present model, the muon is assumed to tunnel under a potential barrier $H=190 \mathrm{meV}$. The use of $b=1.5 \AA$ produces $\beta=6.8 \times 10^{-5}$ yielding the jump rate formula

$$
\frac{1}{\tau}=10^{5} T^{3 / 2} \mathrm{e}^{-2200 / T} \quad\left[\mathrm{~s}^{-1}\right] .
$$

This formula closely follows the experimental results over $220-300 \mathrm{~K}$.

\section{5. $V_{3} S i$}

Muon diffusion in this high temperature superconducting alloy has been studied over $2-350 \mathrm{~K}$ [15]. In this alloy muon is found to be localized below $200 \mathrm{~K}$. 
Around $200 \mathrm{~K}$ the muon starts to diffuse in a tumnelling process and over the temperature range $200-350 \mathrm{~K}$, the jump rate for muon diffusion is given [16] by

$$
\frac{1}{\tau}=(6 \pm 2) \times 10^{10} \mathrm{e}^{-(2550 \pm 220) / T} \quad\left[\mathrm{~s}^{-1}\right] \text {. }
$$

In our present model the muon is assumed to tunnel with an activation energy $2550 \mathrm{~K}$. With $b=0.9 \AA, \beta$ becomes $2 \times 10^{-3}$ yielding the jump rate formula

$\frac{1}{\tau}=2 \times 10^{7} T^{3 / 2} \mathrm{e}^{-2550 / T} \quad\left[\mathrm{~s}^{-1}\right]$.

This formula is a good fit to the experimental results over $200-350 \mathrm{~K}$.

\section{Discussions}

The present theory for muon diffusion can be extended to other good targets over suitable temperature ranges of temperatures.

For $\mathrm{V}_{3}$ Si the present theory yields $\beta=2 \times 10^{-3}$ which is very close to the experimental value $6 \times 10^{-3}$.

The author is much gratcful and indebted to Prof. D.K. Sinha, Calcutta University, for stimulating inspirations and many valuable suggestions on the problem.

\section{References}

[1] H. Sarkar, G.C. Mahato, Acta Phys. Pol. A 70, 403 (1986).

[2] G.C. Mahato, Acta Phys. Pol. A 72, 789 (1987).

[3] G.C. Mahato, Acta Phys. Pol. A 77, 597 (1990).

[4] G.C. Mahato, Acla Phys. Pol. A 78, 535 (1990).

[5] G.C. Mahato, Acla Phys. Pol. A 85, 937 (1994).

[6] V.G. Nosov, I.V. Takovleva, Nucl. Phys. 68, 609 (1965).

[7] A. Schenck, Muon Rolation Spectroscopy, Adam Hilger Ltd., Bristol 1985, p. 251.

[8] S.F.J. Cox, J. Phys. C., Solid Slate Phys. 20, 63 (1987).

[9] P. Bandyopadhyay, Lelt. Nuovo Cimento Ser. I 3, 437 (1970).

[10] V.G. Grebinnik, I.I. Gurevich, V.A. Zlıukov, A.P. Manych, E.A. Meleshko, L.A. Muratova, B.A. Nikolskii, V.I. Selivanov, V.A. Suetin, Sov. Phys. JETP 41, 777 (1975).

[11] O. Hartmann, Hyperfine Interact. 64, 641 (1990).

[12] O. Hartmann, S.W. Harris, R. Wappling, Phys. Scr. 45, 402 (1992).

[13] R. Hampelmann, D. Richter, O. IIartmann, E. Karlsson, R. Wappling, J. Chem. Phys. 90, 1935 (1989).

[14] J. Shinar, D. Davidov, D. Shaltiel, Phys. Rev. D 30, 6331 (1984).

[15] A. Baudry, P. Boyer, L.P. Ferreira, S.W. Harris, S. Miraglia, L. Pontonnier, J. Phys., Condens. Malter 4, 5025 (1992).

[16] A. Yaouance, O. Hartmann, E. Karlsson, E. Wakelgard, R. Wappling, D. Fruchart, R. Fruchart, J.P. Senateur, Hyperfine Inleract. 31, 93 (1986). 